\title{
Piezoelectric Nanofunctionality Integratedinto Advanced Textiles for Energy Harvesting
}

\author{
Valeria Suarez Vega ${ }^{1}$, Karen Lozano ${ }^{2}$, Okenwa Okoli ${ }^{3}$ and Mohammed J Uddin ${ }^{1 *}$ \\ ${ }^{1}$ Department of Chemistry, The University of Texas Rio Grande Valley, US \\ ${ }^{2}$ Department of Mechanical Engineering, The University of Texas Rio Grande Valley, US \\ ${ }^{3}$ Department of Industrial \& Manufacturing Engineering, FAMU-FSU College of Engineering, US
}

Submission: January 17, 2020; Published: March 09, 2020

*Corresponding author: Mohammed J Uddin, Photonics and Energy Research Laboratory (PERL), Department of Chemistry, The University of Texas Rio Grande Valley, Edinburg TX 78539, US

Keywords: Nanofunctionality; Convenient energy; Biomechanical movements; Mechanical strain; Functional textiles; Piezoelectric; Wearable generator field; Power electronics

\section{Mini Review}

The fast development of low power electronics demands for the invention of convenient energy sources. A promising solution to this new problem is the harvesting of energy from biomechanical movements. This mini review presents the basics to an alternative way of harvesting energy from mechanical strain through the piezoelectric effect. Some recent advances on functional textiles are presented and in the concluding section possible applications and challenges to the wearable generator field are discussed. The rapid development and availability of low-power electronics demands for the invention of compact and convenient energy sources. A promising solution to this matter is the use of functional textiles to harvest energy from biomechanical movements [1]. Functional textiles are textiles that can produce a practical outcome when there is a detectable change in their surroundings. The resultant response could be chemical, electric, thermal or some other type [2]. In this review some smart textiles and their ability to convert a mechanical stimulus into electrical energy will be discussed in addition to some possible applications for their energy harvesting capabilities.

\section{Piezoelectric Textiles}

First, let's talk about piezoelectricity and how it can be applied to textiles. The piezoelectric effect is the ability of certain materials to generate an electric charge in response to an applied mechanical strain. The basic principle is that a change in the molecular structure of the piezoelectric material causes the formation of electrical dipoles due to localized charge separation [2]. When the piezo potential is formed between the electrodes and coupled to an external load, the potential will move electrons through the external circuit in order to achieve a new equilibrium [3]. Based on this idea, the piezoelectric effect can be used as a simple solution for mechanical energy harvesting from fully textile generators. There is a wide range of piezoelectric materials like ceramics, polymers, and composites; However, the most promising type of materials for their incorporation into textiles are piezopolymers due to their low weight, flexibility and strength. Textile based nanogenerators are divided into three groups; single-fiber based, fabric-based with textile forming structures, and fabric-based with multilayer stacking structures [3]. Single-fiber based nanogenerators present coaxial and core-shell structures formed by different materials. Their piezoelectric outputs are low due to the small number of active areas. One promising strategy is to combine multiple fibers into three- and two-dimensional fabrics using various textile forming structures. Fabric-based piezoelectric nanogenerators can be obtained by traditional textile forming processes as well as from planar fabric substrates and nanofiber webs [3].

There are various methods used to test the output of piezoelectric materials depending on their intended use. Nevertheless, one of the most common test methods for energy harvesting is impact testing. Impact testing consists on measuring 
possible power outputs from force pulses produced in a range of frequencies [4].

\section{Advances}

The first nanogenerator was developed in 2006 by Wang \& Song [5]. Their device exploited the piezoelectric effect generated by the bending of an aligned array of $\mathrm{ZnO}$ nanowires which evidentiated the potential conversion of mechanical vibrations into electrical energy. Since Wang and Song's development there has been intense research on different piezoelectric devices, materials and structures. An energy generating piezoelectric textile where the power output is enhanced by water was developed by Lund et al in 2018 [1]. The output generated by this melt-spun continuous microfiber was $3.5 \mathrm{~V}$ and demonstrated practical energy harvesting by its integration into a shoulder strap from a laptop case [1]. In Figure 1, the integration of this fiber into the case strap can be observed and the output generated when going up and down stairs. The intrinsic crystal structure of a material dictates the piezoelectric coefficient which correlates with its ability to reversibly convert mechanical to electrical energy. However, functionalized lead zirconate titanate (PZT) nanoparticle colloids have been sculpted into 3D forms through additive manufacturing allowing for the rational design of electrical-mechanical anisotropy and orientation effects. This work can be considered as a step closer to the logical design of piezoelectric transductors [6].

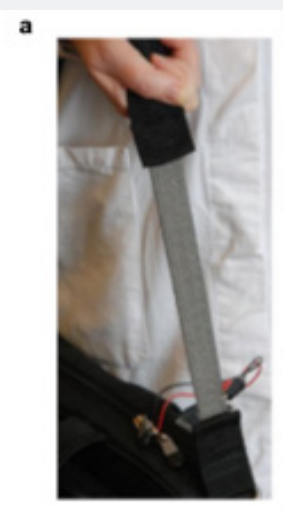

b

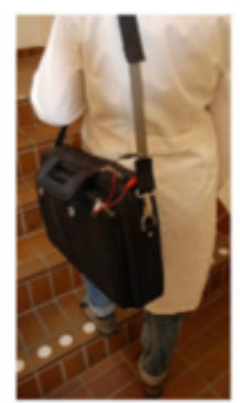

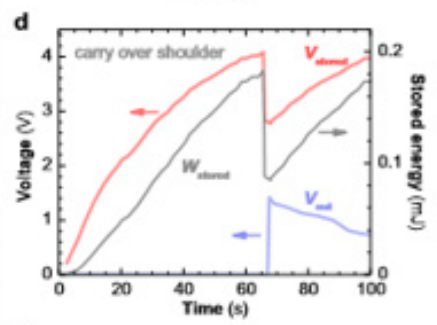

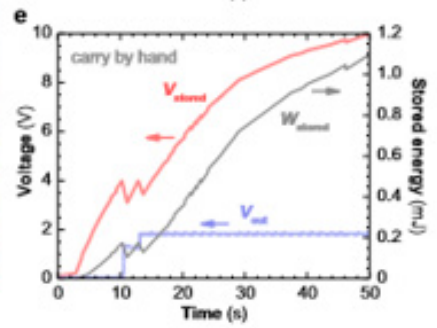

Figure 1: Energy harvesting with a piezoelectric shoulder strap.

a) A twill-woven textile band with PAsilver conducting yarn as the outer electrode was sewn into the shoulder strap of a laptop case.

b) Walking up and down stairs with the laptop bag over the shoulder generates a c piezoelectric voltage where the peaks correspond to footfall.

Cheng et al. [7] proposed a triboelectric-electromagneticpiezoelectric hybrid energy harvester based on rotational motion which exhibits satisfactory outputs. They reported that at a rotational speed of $45 \mathrm{rpm}$, the hybrid harvester produces an opencircuit voltage of $206 \mathrm{~V}(202 \mathrm{~V})$, a short-circuit current of $3.1 \mu \mathrm{A}$ $(3.2 \mu \mathrm{A})$ and transferred charges of $91 \mathrm{nC}(92 \mathrm{nC})$. [7] Their hybrid energy harvester charged a commercial capacitor to $10 \mathrm{~V}$ in about
150 s and that demonstrates the ability of this energy harvester to supply energy for low-power electronic devices.

\section{Conclusion and Perspectives}

The purpose of this report is to present a brief summary into the use of the piezoelectric effect in fibers and summarize recent advancements on textile generators. One of the goals for this 
field is to exploit this effect and incorporate these piezoelectric nanogenerators into everyday life. The applications of textilebased generators are endless, but a representative area is wearable electronics. Wearable electronics are unobstructive devices worn by a person and aid with human life such as communicating, interacting, monitoring and sensing [8]. Even though the applications of piezoelectric generators are endless, there still is a great gap between the lab setting and the practical applications. Piezoelectric fibers have been integrated into laptop cases [1] and they have demonstrated their capability to charge a commercial capacitor in a matter of seconds [7], but there still is a long way to commercial applications. The main challenges that are needed to overcome are wearability, stability, output, scalability and the cost effectiveness fabrication of these functional textiles. All these unresolved issues have hindered the development, but it is firmly believed that many issues will be solved, and textile generators will become part of the everyday life.

\section{References}

1. Lund A, Rundqvist K, Nilsson E, Yu L, Hagström B, et al. (2018) Energy Harvesting Textiles for a Rainy Day: Woven piezoelectrics Based on Melt-Spun PVDF macrofibres with a Conducting Core. npj Flexible Electronics 2 (1): 1-9.
2. Waqar S, Wang L, John S (2015) Piezoelectric Energy Harvesting from Intelligent Textiles. Electronic Textiles, pp. 173-197.

3. Dong K, Peng X, Wang ZL (2019) Fiber/Fabric-Based Piezoelectric and Triboelectric Nanogenerators for Flexible/Stretchable and Wearable Electronics and Artificial Intelligence. Advanced Materials 32(5): e1902549.

4. Zeng W, Tao XM, Chen S, Shang S, Chan HLW, Choy SH (2013) Highly Durable All-Fiber Nanogenerator for Mechanical Energy Harvesting. Energy \& Environmental Science 6(9): 2631-2638.

5. Wang ZL, Song JH (2006) Piezoelectric Nanogenerators Based on Zinc Oxide Nanowire Arrays. Science 312(5771): 242-246.

6. Cui H, Hensleigh R, Yao D, Maurya D, Kumar P, et al. (2019) ThreeDimensional Printing of Piezoelectric Materials with Designed Anisotropy and Directional Response. Nature Materials 18(3): 234241.

7. Ma T, Gao Q, Li Y, Wang Z, Lu X, et al. (2019) An Integrated TriboelectricElectromagnetic-Piezoelectric Hybrid Energy Harvester Induced by a Multifunction Magnet for Rotational Motion. Advanced Engineering Materials 22(2): 1900872.

8. X Tao (2005) Wearable Electronics and Photonics. ( $1^{\text {st }}$ edn), Woodhead Publishing, UK, pp. 1-256.
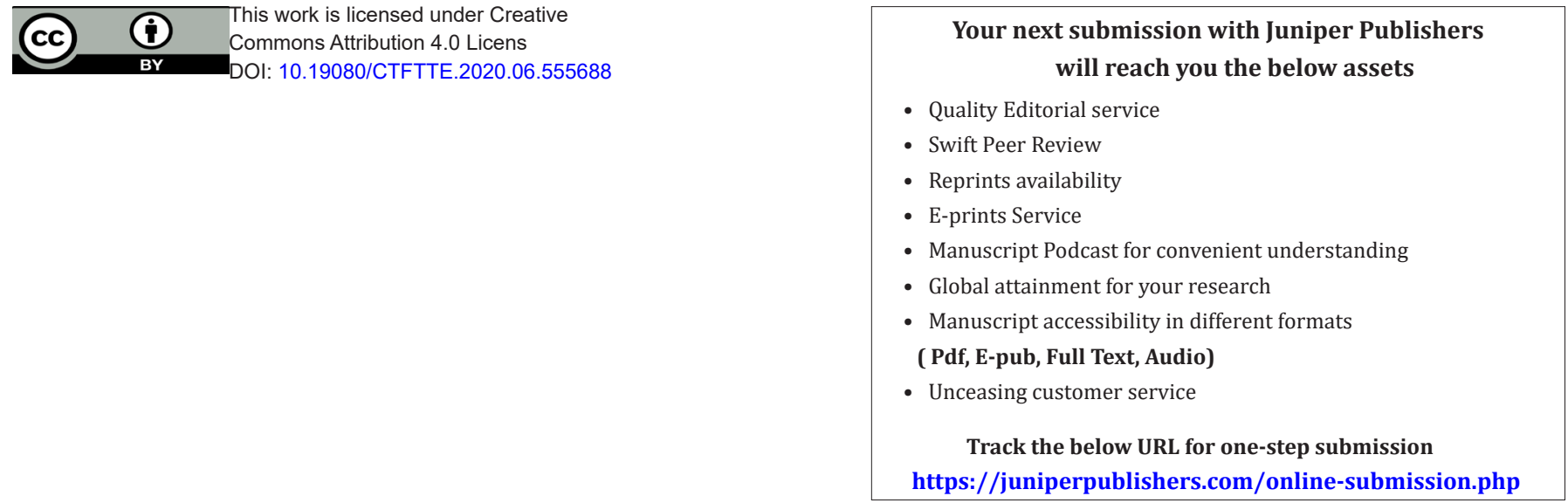